\title{
Evaluation of a flat-field grazing incidence spectrometer for highly charged ion plasma emission in soft $x$-ray spectral region from 1 to $10 \mathrm{~nm}$
}

Thanh Hung Dinh, Yoshiki Kondo, Toshiki Tamura, Yuichi Ono, Hiroyuki Hara, Hiroki Oikawa, Yoichi

Yamamoto, Masahiko Ishino, Masaharu Nishikino, Tetsuya Makimura, Padraig Dunne, Gerry O'Sullivan,

Shigeru Ohta, Ken Kitano, Takeo Ejima, Tadashi Hatano, and Takeshi Higashiguchi

Citation: Review of Scientific Instruments 87, 123106 (2016); doi: 10.1063/1.4971421

View online: $\mathrm{http}: / / d x$. doi.org/10.1063/1.4971421

View Table of Contents: http://aip.scitation.org/toc/rsi/87/12

Published by the American Institute of Physics

\section{Articles you may be interested in}

Publisher's Note: "Evaluation of a flat-field grazing incidence spectrometer for highly charged ion plasma emission in soft x-ray spectral region from 1 to $10 \mathrm{~nm}$ " [Rev. Sci. Instrum. 87, 123106 (2016)]

Review of Scientific Instruments 88, 029902029902 (2017); 10.1063/1.4973926

Measurement of the electro-optic coefficient during the photoelectric-field assisted poling using a MachZehnder interferometer

Review of Scientific Instruments 87, 123102123102 (2016); 10.1063/1.4969057

Characterization of electrical noise limits in ultra-stable laser systems

Review of Scientific Instruments 87, 123105123105 (2016); 10.1063/1.4971852

Opto-electro-modulated transient photovoltage and photocurrent system for investigation of charge transport and recombination in solar cells

Review of Scientific Instruments 87, 123107123107 (2016); 10.1063/1.4972104

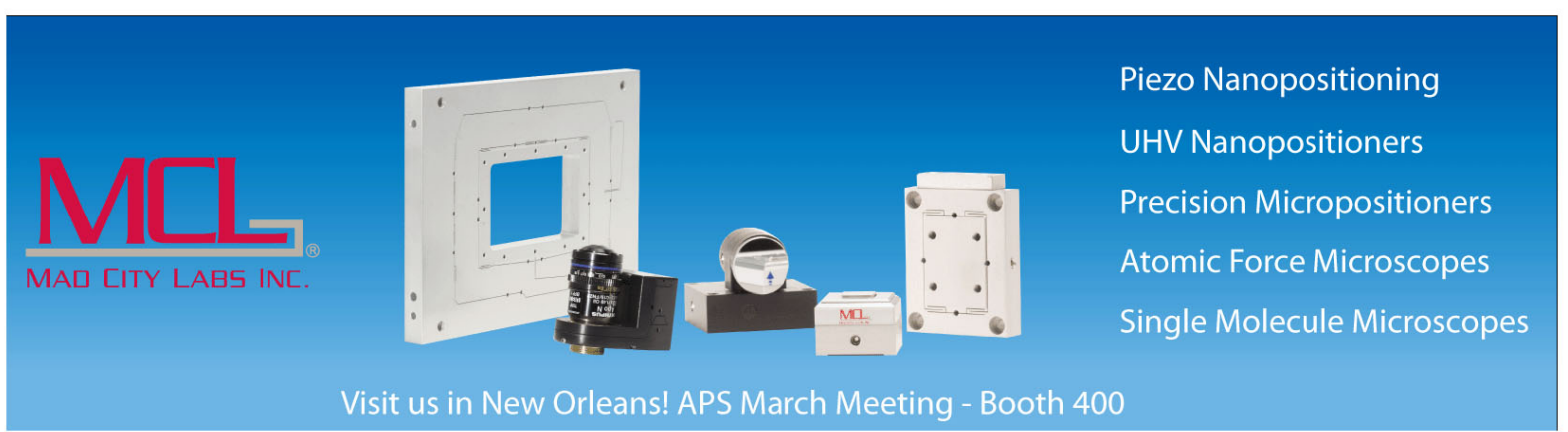




\title{
Evaluation of a flat-field grazing incidence spectrometer for highly charged ion plasma emission in soft $\mathrm{x}$-ray spectral region from 1 to $10 \mathrm{~nm}$
}

\author{
Thanh Hung Dinh, ${ }^{1,2, a)}$ Yoshiki Kondo, ${ }^{1}$ Toshiki Tamura, ${ }^{1}$ Yuichi Ono, ${ }^{1}$ Hiroyuki Hara, ${ }^{1}$ \\ Hiroki Oikawa, ${ }^{1}$ Yoichi Yamamoto, ${ }^{2}$ Masahiko Ishino, ${ }^{2}$ Masaharu Nishikino, ${ }^{2}$ \\ Tetsuya Makimura, ${ }^{3}$ Padraig Dunne, ${ }^{4}$ Gerry O'Sullivan, ${ }^{4}$ Shigeru Ohta, ${ }^{5}$ Ken Kitano, ${ }^{5}$ \\ Takeo Ejima, ${ }^{6}$ Tadashi Hatano, $^{6}$ and Takeshi Higashiguchi ${ }^{1, b)}$ \\ ${ }^{1}$ Department of Electrical and Electronic Engineering, Faculty of Engineering and Center for Optical Research \\ and Education (CORE), Utsunomiya University, 7-1-2 Yoto, Utsunomiya, Tochigi 321-8585, Japan \\ ${ }^{2}$ Kansai Photon Science Institute, National Institutes for Quantum and Radiological Science and Technology \\ (QST), 8-1-7 Umemidai, Kizugawa, Kyoto 619-0215, Japan \\ ${ }^{3}$ Institute of Applied Physics, University of Tsukuba, Tsukuba, Ibaraki 305-8573, Japan \\ ${ }^{4}$ School of Physics, University College Dublin, Belfield, Dublin 4, Ireland \\ ${ }^{5}$ Vacuum and Optical Instruments, 2-18-18 Shimomaruko, Ohta-ku, Tokyo 146-0092, Japan \\ ${ }^{6}$ Institute of Multidisciplinary Research for Advanced Materials, Tohoku University, 2-1-1 Katahira, Aoba-ku, \\ Sendai 980-8577, Japan
}

(Received 27 May 2016; accepted 21 November 2016; published online 20 December 2016; corrected 27 December 2016)

\begin{abstract}
A flat-field grazing incidence spectrometer operating on the spectral region from 1 to $10 \mathrm{~nm}$ was built for research on physics of high temperature and high energy density plasmas. It consists of a flat-field grating with 2400 lines/mm as a dispersing element and an x-ray charged coupled device (CCD) camera as the detector. The diffraction efficiency of the grating and the sensitivity of the CCD camera were directly measured by use of synchrotron radiation at the BL-11D beamline of the Photon Factory (PF). The influence of contamination to the spectrometer also was characterized. This result enables us to evaluate the absolute number of photons in a wide range wavelength between 1 and $10 \mathrm{~nm}$ within an acquisition. We obtained absolutely calibrated spectra from highly charged ion plasmas of $\mathrm{Gd}$, from which a maximum energy conversion efficiency of $0.26 \%$ was observed at a Nd:YAG laser intensity of $3 \times 10^{12} \mathrm{~W} / \mathrm{cm}^{2}$. Published by AIP Publishing. [http://dx.doi.org/10.1063/1.4971421]
\end{abstract}

\section{INTRODUCTION}

Interest in highly charged ion (HCI) spectroscopy has increased in the last decade due in part to the development of efficient and powerful extreme ultraviolet (EUV) and soft $\mathrm{X}$-ray (SXR) sources for applications in x-ray microscopy, ${ }^{1}$ diffractive imaging, ${ }^{2}$ absorption spectroscopy, ${ }^{3}$ and EUV lithography. ${ }^{4}$ Laser-produced HCI plasmas are potentially suitable as laboratory scale high power sources, in which the use of intense unresolved transition arrays (UTAs), instead of discrete line emission, ${ }^{5}$ with reflective rather than transmissive optics has been proposed. Ideally the UTA emission should lie within the reflectance bandwidth of a multilayer mirror. The in-band high-energy emission is attributable, in some cases, to hundreds of thousands of near-degenerate resonance lines lying within a narrow wavelength range. Despite the fact that ideally UTA emission cannot be resolved by a spectrometer, their spectroscopy plays an important role for further understanding of the processes occurring and the physics of high temperature and high energy density plasmas. ${ }^{6}$

One important example of an EUV source is the laserproduced tin $(\mathrm{Sn})$ plasma which has been the exclusive focus of research on $13.5 \mathrm{~nm}$ emission for the next generation

\footnotetext{
a)dinh.thanhhung@qst.go.jp

b)higashi@cc.utsunomiya-u.ac.jp
}

photolithography of semiconductor microchips with feature sizes of $22 \mathrm{~nm}$ and below, in high volume manufacturing (HVM). ${ }^{7,8}$ The choice of wavelength is based on the availability of the Mo/Si multilayer mirrors with a peak reflective coefficient of around 70\% within a narrow bandwidth (BW) of $2 \%$ at a wavelength of $13.5 \mathrm{~nm}$. Recent progress in source development at $13.5 \mathrm{~nm}$ has resulted in an average power of $92 \mathrm{~W}$ recorded at an intermediate focus. ${ }^{9,10}$ Research has already commenced on the development of shorter wavelength sources for production of more highly integrated circuits and other applications. EUV emission at the candidate wavelength of $6 . x \mathrm{~nm}$ has also been identified as especially useful when coupled with a $\mathrm{La} / \mathrm{B}_{4} \mathrm{C}$ multilayer mirror with a realized reflectivity coefficient of around $50 \%$ within a $\mathrm{BW}$ of $0.6 \% .^{11,12}$ EUV sources at $6 . x \mathrm{~nm}$ are provided by laserproduced plasmas (LPPs) of the rare-earth elements Gd and $\mathrm{Tb},{ }^{13,14}$ and other elements, such as Mo and $\mathrm{Kr}^{11,12,15}$ The use of HCI plasmas also has been proposed for laboratory scale SXR sources in the water window region $(\lambda=2.3-4.4 \mathrm{~nm})$ for a compact microscope with a single-shot flash imaging capability. ${ }^{16}$ From the source development point of view, a key important factor is the enhancement of the output energy and energy conversion efficiency by optimizing the plasma parameters for specific applications.

A flat-field grazing incidence spectrograph is a very convenient tool to study the soft $\mathrm{x}$-ray emission from LPPs 
or other sources. The dispersing optic is a concave reflection grating with variable spacing between the grooves that was designed to image the spectrum on a flat plane instead of on the Rowland circle. ${ }^{17-19}$ Thus, flat detectors such as a charge coupled detector (CCD), streak camera, and microchannel plate can be used as recording devices. In a recent development, gratings fabricated by holographic technology demonstrated excellent suppression of higher-order light and stray light compared with mechanically ruled types..$^{20,21} \mathrm{In}$ term of high resolving power, gratings with 1200 lines $/ \mathrm{mm}$ and 2400 lines $/ \mathrm{mm}$ gratings have been recommended for use in the spectral region from 5-40 $\mathrm{nm}$ and $1-6 \mathrm{~nm}$, respectively. Therefore, the 2400 lines/mm grating was only characterized for the wavelength below $6 \mathrm{~nm} .{ }^{20-23}$ For HCI plasma research, LPPs of high- $Z$ elements with $Z=60-83$ produce intense UTA emission between $1 \mathrm{~nm}$ and $10 \mathrm{~nm}$, whose peak wavelengths follow a quasi-Moseley's law. ${ }^{5}$ The diagnostics of these plasmas requires absolute intensity information of the emissions involved over a wide range wavelengths, from which the plasma parameters such as ion charge state and temperature can be determined. A spectrometer employed the both of two gratings is usually used to cover all the wavelength range. However a drawback of this scheme is it takes several processes to obtain the spectral data at round the overlap wavelength region between the two gratings.

In this study, we proposed the use of a holographic fabricated grating with 2400 lines/mm for the spectral region between $1 \mathrm{~nm}$ and $10 \mathrm{~nm}$. A flat-field grazing incidence spectrometer (GIS) was built to investigate the SXR emission from laser-produced HCI plasmas. Measurements of the diffraction efficiency of the grating and the response of an $\mathrm{x}$-ray CCD camera using synchrotron radiation at the BL-11D beamline of the Photon Factory (PF) enable us to evaluate the absolute number of photons in a wide range wavelength below $10 \mathrm{~nm}$ within an acquisition. Finally, we demonstrated the calibrated SXR spectra from Gd plasmas produced by a $\mathrm{Nd}$ :YAG laser.

\section{SETUP}

The schematic setup of the GIS for LPP experiments is presented in Fig. 1. Starting from the source, it consists of a pair of a bilateral continuously adjustable slits, a goldcoated-concave reflection grating with 2400 lines $/ \mathrm{mm}$, and a detector. The grating was provided by Shimadzu Corporation. The dimensions between the slits, grating, and detector are the same as those described in Kita et al. ${ }^{18}$ To achieve a high signal-to-noise ratio, the slit was set with a width of

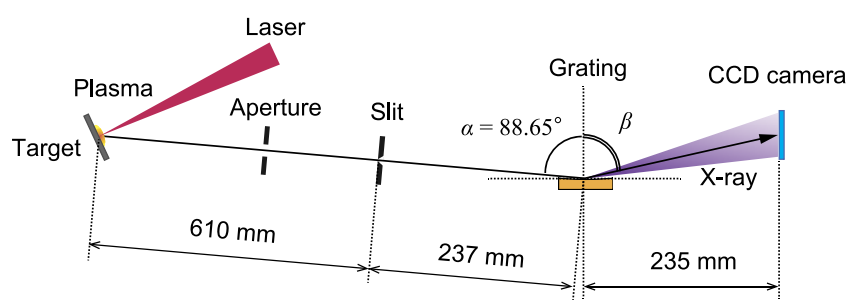

FIG. 1. Schematic diagram of the flat-field grazing incident spectrometer.
$100 \mu \mathrm{m}$. The x-ray detector was a back-illuminated CCD camera provided by Andor. It was placed in the focal plane of the reflection grating, and was air cooled to reduce noise. The CCD consists of $2048 \times 2048$ pixels (each pixel has dimensions $13.5 \times 13.5 \mu \mathrm{m}^{2}$ ) and was directly exposed to the SXR radiation. It was mounted on a two dimensional linear stage. One dimension allows the surface of the CCD array to be positioned in the focal plane of the grating, by adjusting the distance from the grating, while movement in the other dimension is used to select the wavelength range. A mask was placed in front of the CCD that blocked the zero order and stray light from the grating. An aperture with a diameter of about $2 \mathrm{~mm}$ was placed behind the plasma source to prevent the spectrometer from debris and also reduce scattered light. Due to the small number of components involved, a low optical loss is achieved. Thus, a single shot spectrograph can be obtained with the GIS.

A 150-ps Nd:YAG laser with a maximum pulse energy of $250 \mathrm{~mJ}$ at $\lambda_{L}=1064 \mathrm{~nm}$ and an 8-ns Nd:YAG laser giving the maximum pulse energy of $1 \mathrm{~J}$ at $\lambda_{L}=1064 \mathrm{~nm}$ were employed to provide the desired variation of laser intensity. The laser beam was incident normally onto planar high- $Z$ metal targets in vacuo. The expected focal spot size, produced by an anti-reflection coated plano-convex BK7 lens with a focal length of $30 \mathrm{~cm}$, had a full width at half-maximum (FWHM) of approximately $50 \mu \mathrm{m}$. The laser was operated in single shot mode. The GIS was positioned at $30^{\circ}$ with respect to the incident laser axis. The pressure inside the GIS chamber was $5 \times 10^{-4} \mathrm{~Pa}$ during the measurement.

\section{CALIBRATION OF THE SPECTROMETER}

In order to determine the photon number of SXR emitted from HCI plasma sources, knowledge of the GIS's dispersion and absolute response of the spectrometer elements as a function of wavelength are required. The details of the calibration are presented in the following.

\section{A. The dispersion of the GIS}

The dispersion of the GIS was determined by using the well-known line emission from LPPs of targets such as carbon, aluminum, and silicon nitride. The dispersion as a function of position along the image plane of the grating was found by measuring the centers of the $\mathrm{x}$-ray emission lines from each of the LPPs mentioned above, fitting a Gaussian curve to each line to determine its centroid in CCD pixel space, converting the pixel number to distance along the image plane, plotting the wavelength of the lines versus their centroids in millimeters, and fitting the curve. The equation used to fit the curve is the standard grating equation given by

$$
m \lambda=d(\sin \alpha-\sin \beta),
$$

where $m$ is the diffraction order, $d$ the line spacing at the center of the grating, $\alpha$ the incidence angle measured from the normal, $88.65^{\circ}$, and $\beta$ the dispersion angle given by $\tan ^{-1}\left[235 /\left(y-y_{0}\right)\right]$, where $y$ is the spectral line position in millimeters and $y_{0}$ the off-set. A time-integrated spectrum 


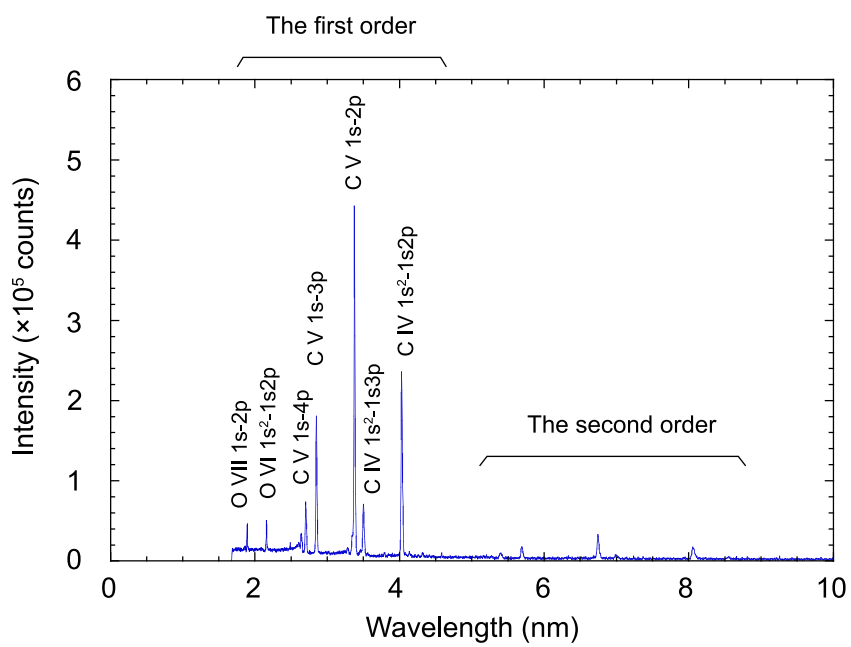

FIG. 2. Typical spectrum of a carbon plasma produced by a 150-ps Nd:YAG laser. A resolving power $\lambda / \Delta \lambda=277$ was achieved at $\lambda=3.37 \mathrm{~nm}$.

of a carbon plasma produced by the 150-ps Nd:YAG laser is displayed in Fig. 2. The spectral lines of both the first and second order diffraction were observed. The wavelength position of these lines was well defined with a discrepancy less than $0.005 \mathrm{~nm}$. The spatial width (FWHM) of the $\mathrm{C}^{5+} 1 s-2 p$ line at $3.37 \mathrm{~nm}$ is $54 \mu \mathrm{m}$, corresponding to a resolving power $\lambda / \Delta \lambda=277$ which is close to the achievement of the previous studies. ${ }^{22,23}$ In contrast, the width of the line itself is expected to be dominated by Doppler broadening, which can be calculated to be $\lambda / \Delta \lambda=2000$. A ratio of the second order to the first order intensity of $10 \%$ was observed at $3.37 \mathrm{~nm}$. Therefore, the effect of the high orders should be eliminated from the acquired spectra for a highly accurate evaluation of UTA emission.

\section{B. Measurement of the gating efficiency}

The diffraction efficiency of the 2400 lines/mm grating was measured by using the soft $\mathrm{x}$-ray reflectometer installed at the BL-11D beamline of the Photon Factory (PF) in Japan. ${ }^{24-26}$ Figure 3(a) indicates a schematic diagram of the measurement arrangement. The grating was located at the center of the reflectometer chamber. A divergent angle less than $0.1^{\circ}$ and a FWHM diameter of approximately $200 \mu \mathrm{m}$ at the focal point of the incident beam were measured by a knife-edge scan as described by Hatano et al. ${ }^{25}$ The diffraction efficiency was derived as the ratio between the incident and diffracted powers. To determine this ratio, the angular profiles of the incident and diffracted beam were scanned by a detector, which consisted of an x-ray photo-diode and a slit with a width of $100 \mu \mathrm{m}$. In other words, this detector was used as a quasi-array sensor. The distance from the grating to the detector was about $255 \mathrm{~mm}$ and was fixed due to the configuration of the reflectometer. Figure 3(b) shows the measured angular profiles of the first, second, and third orders at a wavelength of $3 \mathrm{~nm}$. Scanning position is defined as the angle of $\varphi$ in Fig. 3(a). On both sides of these profiles, the detected signal is close to zero, which implies that no scattered light from the grating could be observed. Figure 4 depicts the grating efficiency as a function
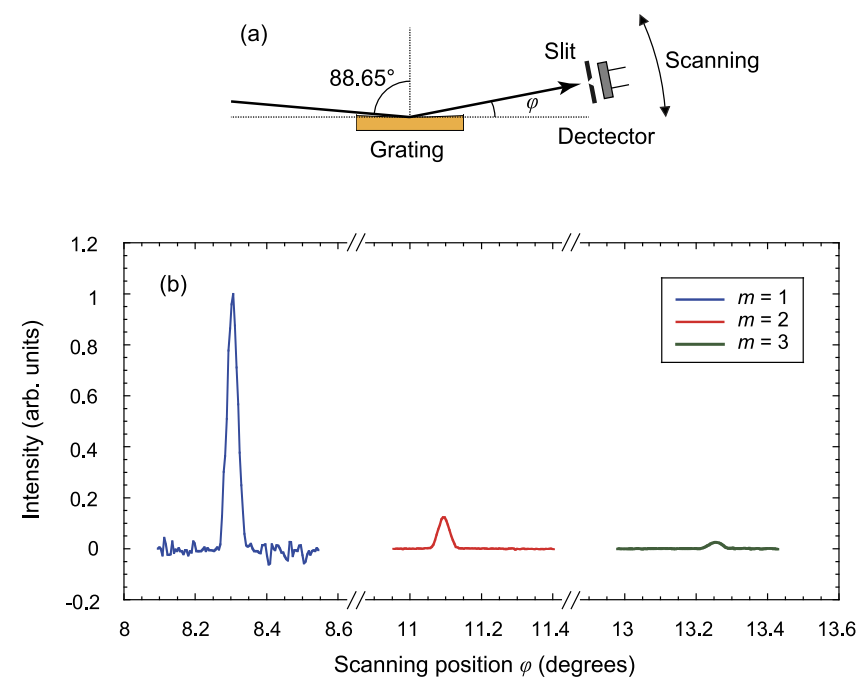

FIG. 3. (a) Experimental setup to measure the diffraction efficiency of the grating. (b) The angular profile of the first, second, and third orders of the diffracted beam at a wavelength of $3 \mathrm{~nm}$. The distance from the grating to the detector was about $255 \mathrm{~mm}$.

of the incident wavelength. A first order diffraction efficiency exceeding $3 \%$ was observed for the wavelength region around $\lambda=2.75 \mathrm{~nm}$ and $\lambda>8.0 \mathrm{~nm}$. This result is higher than that from a previous report of about $2 \% .{ }^{21}$ One of the reasons for this difference may be improvements in the processing technology of the grating. At a wavelength of $3.37 \mathrm{~nm}$, the ratio of second to first order is estimated to be $11 \%$. This result is similar to that measured by using the spectrum of a carbon LPP in Fig. 2. The presence of dips in the short wavelength region could be observed in both first and second order spectra. These dips are caused by $K$-absorption of oxygen and carbon originating from hydrocarbon contamination on the surface of the grating. We expect that diffraction efficiency at a wavelength of $4.25 \mathrm{~nm}$ dropped $30 \%$, corresponding to the thickness of the contamination of about $15 \mathrm{~nm}$. It should be noted that our spectrometer has been used in LPP experiments

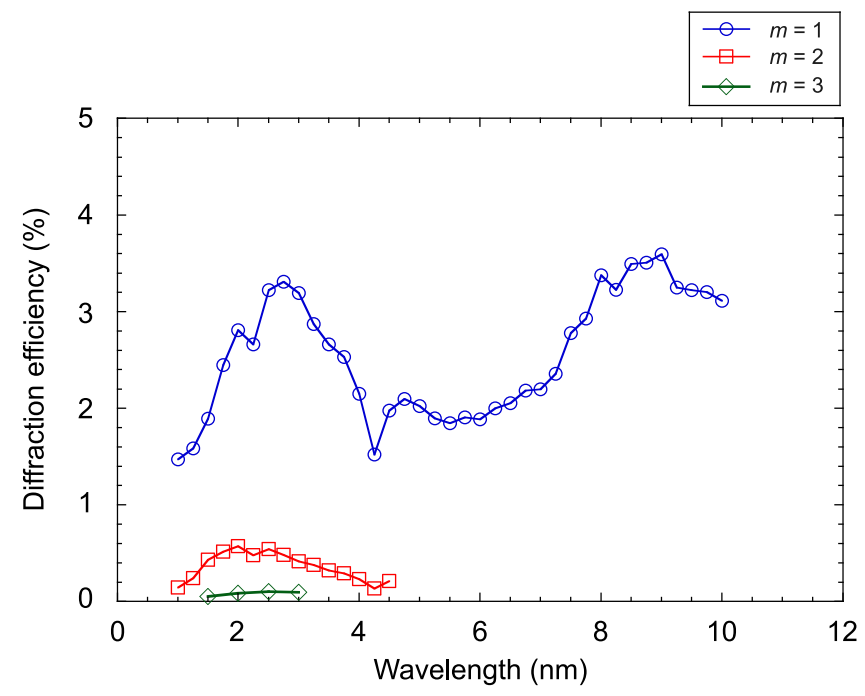

FIG. 4. The diffraction efficiency of the 2400 lines/mm grating as a function of wavelength. 
for more than 5 years. The evaluation of contamination effect is very important in estimating absolute photon flux, particularly in the water- and carbon-window spectral regions.

\section{Spectral response of the CCD camera}

It should be noted that the CCD camera does not record the photon number directly. It records analog to digital unit counts. Therefore, the sensitivity of the CCD camera given in terms of the number of counts per 1 incident photon should be evaluated. The sensitivity of the CCD can be calculated by using an approximate model described in the following. The number of counts $I$ recorded by the CCD camera can be converted to photon number using the formula

$$
N=\frac{\operatorname{Ig} w}{\mathrm{QE}(\lambda) E(\lambda)},
$$

where the gain, $g$, is given by the manufacturer to be 3.7 electrons/count, the quantum efficiency, $\mathrm{QE}(\lambda)$, which is the probability to detect a photon, $w$ is the work function for creating an electron-hole pair in the Si photoactive region of a $\operatorname{CCD}(3.65 \mathrm{eV} /$ electron), and $E$ is the energy of the incident photon in $\mathrm{eV}$. Therefore, we can write the sensitivity $R$ of the CCD camera as

$$
R(\lambda)=\frac{I(\lambda)}{N(\lambda)}=\frac{\mathrm{QE}(\lambda) E(\lambda)}{g w} .
$$

To calculate the quantum efficiency of the CCD chip, we use an approximate model as described in Li et al. and the references therein. ${ }^{27}$ Ideally, a back-illuminated CCD chip consists of a dead layer and an active region. The dead layer is formed by an unavoidable potential which prevents photoelectrons from reaching the active region. The quantum efficiency is the probability of the detection of the photoelectrons in the active layer. In fact, the SXR region is very sensitive to the contamination available on the CCD surface; therefore, the quantum efficiency can be expressed as the following equation:

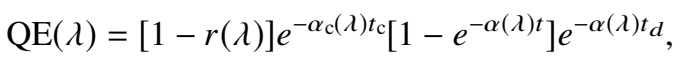

where $r$ is the reflectivity of the CCD surface $(\approx 0$ in our domain), $t, t_{c}$, and $t_{d}$ are the thickness of the active region, the contamination layer, and the dead layer, respectively. $\alpha_{c}$ and $\alpha$ are the absorption coefficients for the contamination and the silicon, respectively.

Measurement of the sensitivity was also performed at the BL-11D beamline of the PF. The experimental setup is shown in Fig. 5(a). To minimize the influence of the impure light included in the beamline, ${ }^{24,26}$ an Al filter with a thickness of $200 \mathrm{~nm}$ was used. A calibrated AXUV100G x-ray diode was used to determine the number of photons incident onto the $\mathrm{x}$-ray $\mathrm{CCD}$ camera. The $\mathrm{CCD}$ was cooled at $-50{ }^{\circ} \mathrm{C}$ with a pressure in the calibration chamber of $5 \times 10^{-5} \mathrm{~Pa}$. Since the high vacuum degree was achieved, influence of the residual contamination such as nitrogen, water vapor, and carbon compounds, which deposit and condense onto the cooled CCD surface, was negligibly small. ${ }^{28}$ This type of contamination usually can be removed by warming up the chip. Figure 5(b) indicates an image recorded by the CCD camera setting at an exposure time of $0.5 \mathrm{~s}$ and a pixel readout rate of $3 \mathrm{MHz}$. (a)

Dectector

Beamline shutter

(CCD camera or photodiode)
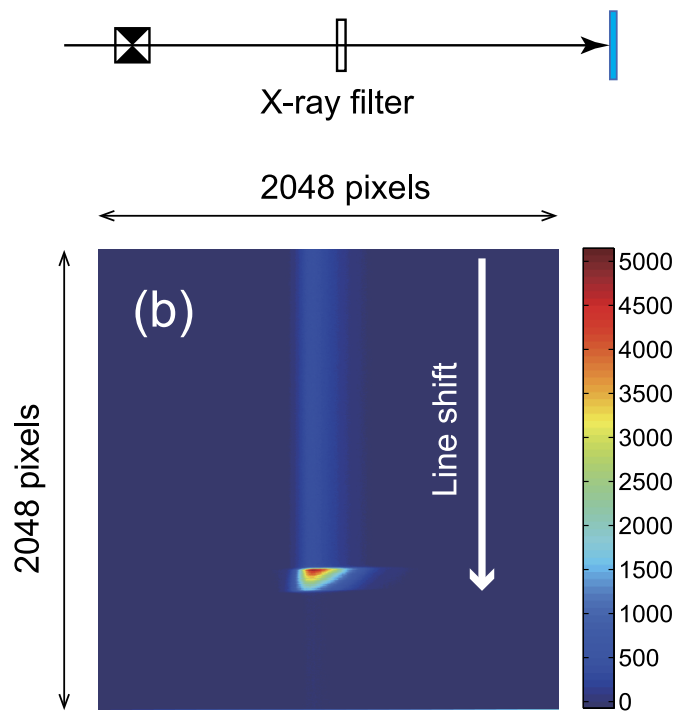

FIG. 5. (a) Experimental setup to measure the sensitivity of the CCD camera. The beamline shutter was opened until the readout finish. (b) indicates a typical image recorded by the CCD camera.

The line shift speed was $38.55 \mu$ s. The beamline shutter was opened until the readout finished. As shown in Fig. 5(b), the high intensity part is dominated by the digital exposure time while the low intensity part is the smearing during the readout time. From the position of the incident beam on the CCD chip, the effective exposure time was calculated to be $1.54 \mathrm{~s}$ with a discrepancy of less than $3 \%$.

Figure 6(a) shows the measured sensitive $R$ from which the quantum efficiency was derived from Eq. (3) as the circles in Fig. 6(b). There are dips around $\lambda=4.3 \mathrm{~nm}$ and $12.3 \mathrm{~nm}$ in the experimental result, corresponding to the $K$-absorption of carbon and the $L$-absorption of silicon, respectively. The thickness, $t_{c}$, of a carbon layer available on the CCD surface, was estimated as the lines in Fig. 6(b). The experimental result was reproduced by the calculation with $t_{c} \approx 10 \mathrm{~nm}$. Such contamination may be originated during the fabrication of a thinned CCD chip or caused by the debris from LPPs, and is difficult to be removed.

\section{Spectral response of the GIS}

The spectral response of the GIS given in terms of the number of $\mathrm{x}$-ray photons per unit solid angle from the source is

$$
\frac{d N}{d \Omega}=\frac{I(\lambda)}{R(\lambda) \eta_{g}(\lambda)} \times \frac{1}{\Delta \Omega},
$$

where $I$ is the number of counts, $\eta_{g}$ the grating efficiency, $\Delta \Omega$ the solid angle of the spectrometer given by

$$
\Delta \Omega=\frac{s h}{d_{s} d_{\mathrm{CCD}}},
$$

where $h$ is the height illuminated on the CCD detector, $s$ the slit width, $d_{s}$ the distance plasma-slit, and $d_{\mathrm{CCD}}$ the distance 

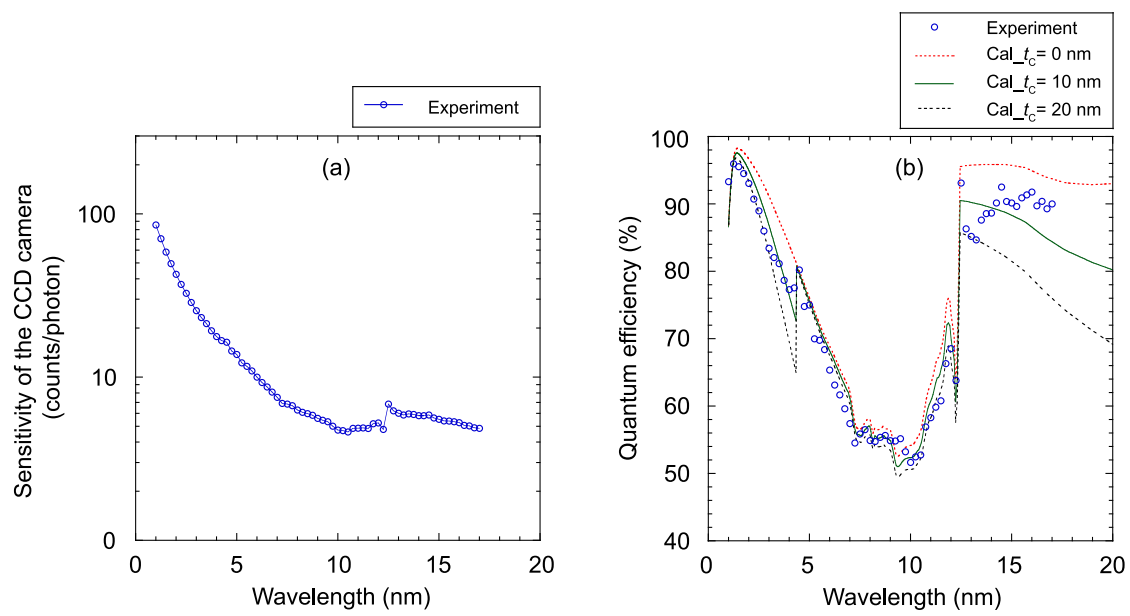

FIG. 6. The sensitivity (a) and the quantum efficiency (b) of the x-ray CCD camera as a function of wavelength. The red dot-, green solid-, and black dash lines in (b) indicate the calculated quantum efficiency with the thickness of carbon contamination layer of $t_{c}=0,10$, and $20 \mathrm{~nm}$, respectively.

plasma-CCD chip. Since the soft $\mathrm{x}$-ray region is very sensitive to the contamination, the response of a spectrometer will change with time. Our evaluation showed that the thickness of the carbon contamination increases about $10-15 \mathrm{~nm}$ after 5 years of use in LPP experiments. In contrast to the CCD camera, the performance of the grating is easily influenced by the contamination. The response change of a spectrometer can be calibrated by comparing the spectral structure of a plasma source with that acquired previously.

\section{SPECTRA OF HCI PLASMA}

In this section, we will discuss the production of an absolutely calibrated spectrum from a HCI plasma. The spectrum recorded by the CCD detector is the convoluted signal of many transitions and can be expressed as the following equation:

$$
H(x)=\int I(\lambda) f(\lambda-x) d \lambda+\varepsilon(x),
$$

where $H$ is the spectrum recorded by the detector, the line shape $f$ is the distribution of $\mathrm{x}$-ray photons along the dispersion axis, and $\varepsilon$ is the noise. As shown in Fig. 2, the noise level in our system is quite low, so it can be neglected. The recorded signal should be deconvolved to give the number of counts for each transition. Furthermore, to produce a highly accurate spectrum, the noise on the CCD camera should be minimized. In the case of the line emission that can be resolved by a spectrometer, the line shape function $f$ can be observed or determined by fitting with Gaussian curves. Consequently, we can find the number of counts $I$ for each transition. However, in the case of UTA emission, since transitions cannot be resolved by a spectrometer, the $\mathrm{x}$-ray count in a specified bandwidth should be estimated. With the assumption that in a narrow bandwidth, the spectrum is continuous and the flux of x-ray photons is similar, the counts recorded on the pixel $i$ of the $\mathrm{CCD}, H_{i}$, can be deconvolved as

$$
I_{i} \approx H_{i} \times \frac{1}{\Delta x} \times \frac{d x}{d \lambda},
$$

where $I_{i}$ is the number of counts of a transition whose center wavelength locate at the center of the pixel $i$, and bandwidth is $\Delta \lambda=(d \lambda / d x) \Delta x, d x / d \lambda$ the linear dispersion of the grating, $\Delta x$ the size of a pixel. Therefore, the photon flux of the source given in terms of the number of photons per unit solid angle, $1 \mathrm{~nm}$ absolute wavelength, and laser pulse interval, is

$$
\frac{d^{2} N}{d \Omega d \lambda}=\frac{I_{i}}{\Delta \lambda} \times \frac{1}{R(\lambda) \eta_{g}} \times \frac{1}{\Delta \Omega}=\frac{H_{i}}{R(\lambda) \eta_{g} \Delta \Omega} .
$$

In Fig. 7, we present time-integrated spectra of the SXR photon flux from an LPP of Gd in which the effect of the second order diffraction was eliminated. The 10-ns Nd:YAG laser was employed to provide laser intensities ranging from $3 \times 10^{11}$ to $3 \times 10^{12} \mathrm{~W} / \mathrm{cm}^{2}$. The UTA emission around a wavelength of $7 \mathrm{~nm}$, wavelength mainly due to $n=4-n=4(\Delta n=0)$ transitions in ions with an open $4 f$ or $4 d$ outermost subshell, increases rapidly with increasing laser intensity. However, the

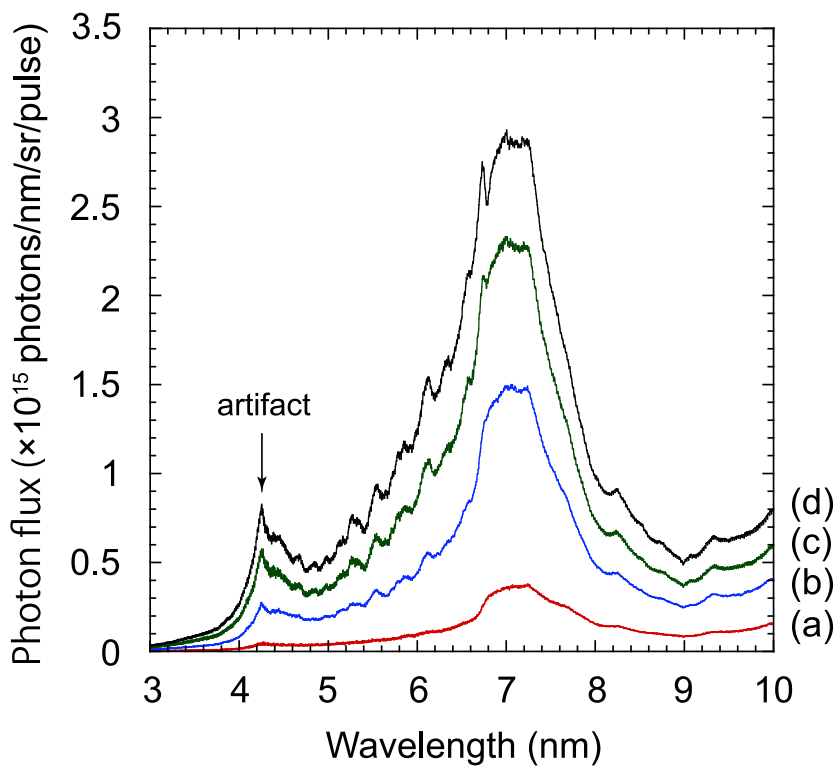

FIG. 7. Absolutely calibrated soft x-ray spectra from Gd HCI plasmas produced by a 10 -ns Nd:YAG laser at laser intensities of $9.7 \times 10^{11}$ (a), $1.9 \times 10^{12}$ (b), $2.6 \times 10^{12}(\mathrm{c})$, and $3.1 \times 10^{12} \mathrm{~W} / \mathrm{cm}^{2}(\mathrm{~d})$. The artifact around $4.2 \mathrm{~nm}$ is caused by the carbon edge. 


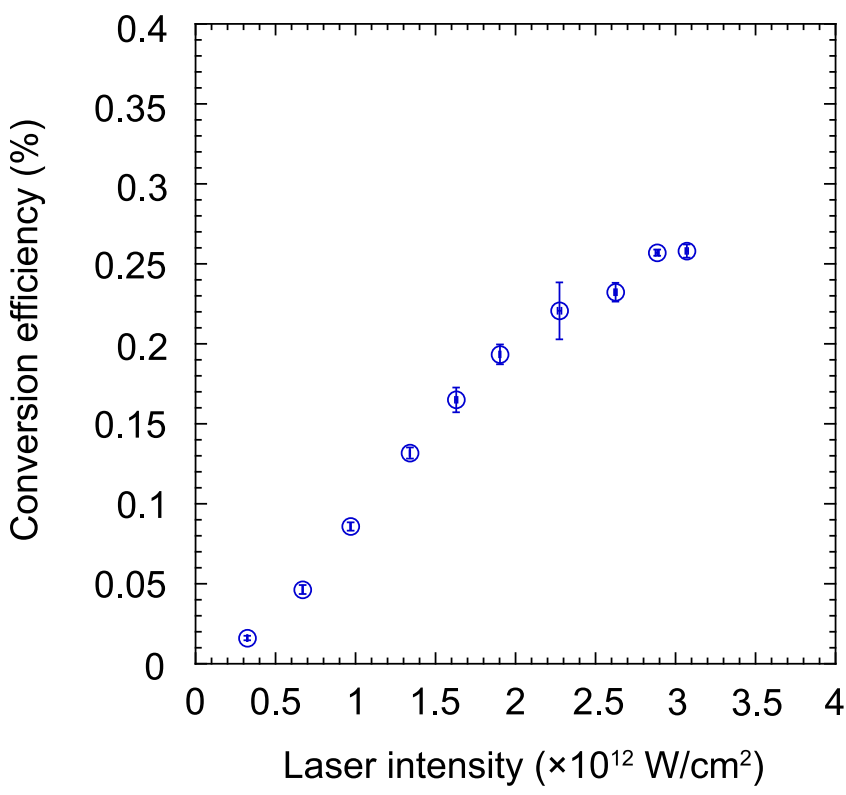

FIG. 8. Laser intensity dependence of conversion efficiency as measured by the GIS. The maximum result of $0.26 \%$ was observed at a laser intensity of $3 \times 10^{12} \mathrm{~W} / \mathrm{cm}^{2}$.

peak wavelength at $7.3 \mathrm{~nm}$ is almost unchanged. The sharp peak at $6.76 \mathrm{~nm}$ and the dip structure below $6.59 \mathrm{~nm}$ first appear at a laser intensity of $2.3 \times 10^{12} \mathrm{~W} / \mathrm{cm}^{2}$.

The energy conversion efficiency at $6.76 \mathrm{~nm}$ in a bandwidth of $0.6 \%$ calculated from the spectra of the Gd plasma is displayed in Fig. 8. It is almost a linear function of the laser intensity in the order of $10^{11} \mathrm{~W} / \mathrm{cm}^{2}$. According to a collisional-radiative (CR) simulation, ${ }^{29}$ the Gd plasma will have strong emission at $6.76 \mathrm{~nm}$ when the plasma temperature is close to $80 \mathrm{eV}$ which optimizes the population of $\mathrm{Gd}^{17+}$ and $\mathrm{Gd}^{18+}$ ions whose resonance emission lies at this wavelength. ${ }^{30}$ Therefore, the increase of the conversion efficiency as shown in Fig. 8 is caused by the increase of the population of these charge states. The large error bar observed at a laser intensity of $2.3 \times 10^{12} \mathrm{~W} / \mathrm{cm}^{2}$ may be caused by the instability at the threshold condition. The maximum conversion efficiency of $0.26 \%$ was observed at a laser intensity of $3 \times 10^{12} \mathrm{~W} / \mathrm{cm}^{2}$.

\section{CONCLUSION}

A simple flat-field grazing incident spectrometer consisting of a holographic fabricated 2400 lines/mm grating and an $\mathrm{x}$-ray CCD camera was built for HCI plasma spectroscopy. The diffraction efficiency of the grating and the absolute response of the CCD camera were directly measured by using the reflectometer installed at the BL-11D beamline of the Photon Factory. This result enables us to evaluate the absolute number of photons in a wide spectral region between $1 \mathrm{~nm}$ and $10 \mathrm{~nm}$ within an acquisition. The influence of contamination to the grating and the CCD camera was characterized. We expected that the thickness of the carbon contamination increases about $10-15 \mathrm{~nm}$ after 5 years of use in LPP experiments. We also studied the calibrated spectra of Gd HCI plasmas which were produced by a Nd:YAG laser. A maximum energy conversion efficiency of $0.26 \%$ was observed at a laser intensity of $3 \times 10^{12} \mathrm{~W} / \mathrm{cm}^{2}$.

\section{ACKNOWLEDGMENTS}

This work was performed under the auspices of MEXT (Ministry of Education, Culture, Sports, Science and Technology, Japan) and "Project for Bio-Imaging and Sensing at Utsunomiya University" from MEXT and was performed under the Cooperative Research Program of "Network Joint Research Center for Materials and Devices" (under Contract Subject No. 2015209) and IMRAM, Tohoku University, Heisei 27 nendo Grant. One of the authors (T.H.D.) also acknowledges support from Grant-in-Aid for Young Scientists B (Grant No. 15K18045) and JSPS Postdoctoral Fellowship for Overseas Researchers (Grant No. P16019). Measurements of the grating efficiency and the response of the $\mathrm{x}$-ray CCD camera were performed under the approval of the Photon Factory Program Advisory Committee (Proposal No. 2015G667). One of the authors (T.H.) also acknowledges support from The Canon Foundation, Research Grant (Basic Research) on TEPCO Memorial Foundation. The UCD group was supported by Science Foundation Ireland International Co-operation Strategic Award No. 13/ISCA/2846.

${ }^{1}$ A. Sakdinawat and A. David, Nat. Photonics 4, 796 (2010).

${ }^{2}$ P. A. C. Takman, H. Stollberg, G. A. Johansson, A. Holmberg, M. Lindblom, and M. Hertz, J. Microsc. 226, 175 (2007).

${ }^{3}$ R. L. Sandberg, A. Paul, D. A. Raymondson, S. Hädrich, D. M. Gaudiosi, J. Holtsnider, R. I. Tobey, O. Cohen, M. M. Murnane, H. C. Kapteyn, C. Song, J. Miao, Y. Liu, and F. Salmassi, Phys. Rev. Lett. 99, 098103 (2007).

${ }^{4}$ C. Peth, F. Barkusky, and K. Mann, J. Phys. D: Appl. Phys. 41, 105202 (2008).

${ }^{5}$ H. Ohashi, T. Higashiguchi, Y. Suzuki, G. Arai, Y. Otani, T. Yatagai, B. Li, P. Dunne, G. O'Sullivan, W. Jiang, A. Endo, H. A. Sakaue, D. Kato, I. Murakami, N. Tamura, S. Sudo, F. Koike, and C. Suzuki, Appl. Phys. Lett. 104, 234107 (2014)

${ }^{6}$ T. H. Dinh, Y. Suzuki, G. Arai, B. Li, P. Dunne, G. O'Sullivan, S. Fujioka, N. Hasegawa, T. Kawachi, M. Nishikino, and T. Higashiguchi, Appl. Phys. Lett. 107, 121101 (2015).

${ }^{7}$ C. Wagner and N. Harned, Nat. Photonics 4, 24 (2010).

${ }^{8}$ V. Y. Banine, K. N. Koshelev, and G. H. P. M. Swinkls, J. Phys. D: Appl. Phys. 44, 253001 (2011).

${ }^{9}$ H. Mizoguchi, H. Nakarai, T. Abe, K. M. Nowak, Y. Kawasuji, H. Tanaka, Y. Watanabe, T. Hori, T. Kodama, Y. Shiraishi, T. Yanagida, G. Soumagne, T. Yamada, T. Yamazaki, S. Okazaki, and T. Saitou, Proc. SPIE 9422, 94220C (2015).

${ }^{10}$ A. A. Schafgans, D. J. Brown, I. V. Fomenkov, R. Sandstrom, A. Ershov, G. Vaschenko, R. Rafac, M. Purvis, S. Rokitski, Y. Tao, D. J. Riggs, W. J. Dunstan, M. Graham, N. R. Farrar, D. C. Brandt, N. Böwering, A. Pirati, N. Harned, C. Wagner, H. Meiling, and R. Kool, Proc. SPIE 9422, 94220B (2015).

${ }^{11}$ Y. Platonov, J. Rodriguez, M. Kries, E. Louis, T. Feigl, and S. Yulin, in Proceedings of 2011 International Workshop on EUV Lithography, Maui, Hawaii, 13-17 June 2011.

${ }^{12}$ E. Louis, I. Makhotkin, E. Zoethout, and S. Müllender, in Proceedings of 2011 International Workshop on EUV and Soft X-ray Sources, UCD, Dublin, Ireland, 7-10 November 2011.

${ }^{13}$ S. S. Churilov, R. R. Kildiyarova, A. N. Ryabtsev, and S. V. Sadovsky, Phys. Scr. 80, 045303 (2009).

${ }^{14}$ T. Otsuka, D. Kilbane, J. White, T. Higashiguchi, N. Yugami, T. Yatagai, W. Jiang, A. Endo, P. Dunne, and G. O’Sullivan, Appl. Phys. Lett. 97, 111503 (2010).

${ }^{15}$ M. Masnavi, J. Szilagyi, H. Parchamy, and M. C. Richardson, Appl. Phys. Lett. 102, 164102 (2013).

${ }^{16}$ T. Higashiguchi, T. Otsuka, N. Yugami, W. Jiang, A. Endo, B. Li, P. Dunne, and G. O'Sullivan, Appl. Phys. Lett. 100, 014103 (2012).

${ }^{17}$ R. J. Fonck, A. T. Ramsey, and R. V. Yelle, Appl. Opt. 21, 2115 (1982). 
${ }^{18}$ T. Kita, T. Harada, N. Nakano, and H. Kuroda, Appl. Opt. 22, 512 (1983). ${ }^{19}$ M. C. Hettrick, J. H. Underwood, P. J. Batson, and M. J. Eckart, Appl. Opt 27, 200 (1988).

${ }^{20}$ T. Yamazaki, E. Gullikson, N. Miyata, M. Koike, Y. Harada, S. Mrowka, U. Kleineberg, J. H. Underwood, M. Yanagihara, and K. Sano, Appl. Opt. 38, 4001 (1999).

${ }^{21}$ M. Koike, K. Sano, E. Gullikson, Y. Harada, and H. Kumata, Rev. Sci. Instrum. 74, 1156 (2003).

${ }^{22}$ A. Saemann and K. Eidmann, Rev. Sci. Instrum. 69, 1949 (1998).

${ }^{23}$ J. Park, G. V. Brown, M. B. Schneider, H. A. Baldis, P. Beiersdorfer, K. V. Cone, R. L. Kelley, C. A. Kilbourne, E. W. Magee, M. J. May, and F. S Porter, Rev. Sci. Instrum. 81, 10E319 (2010).
${ }^{24}$ T. Hatano and S. Aihara, J. Phys.: Conf. Ser. 425, 152018 (2013).

${ }^{25}$ T. Hatano, S. Aihara, K. Uchida, and T. Tsuru, J. Phys.: Conf. Ser. 463, 012010 (2013).

${ }^{26}$ T. Hatano and T. Harada, J. Electron Spectrosc. Relat. Phenom. 196, 156 (2014).

${ }^{27}$ Y. Li, G. D. Tsakiris, and R. Sigel, Rev. Sci. Instrum. 66, 80 (1995).

${ }^{28}$ L. Poletto, A. Boscolo, and G. Tondello, Appl. Opt. 38, 29 (1999).

${ }^{29}$ D. Colombant and G. F. Tonon, J. Appl. Phys. 44, 3524 (1973).

${ }^{30}$ T. Higashiguchi, B. Li, Y. Suzuki, M. Kawasaki, H. Ohashi, S. Torii, D. Nakamura, A. Takahashi, T. Okada, W. Jiang, T. Miura, A. Endo, P. Dunne, G. O’Sullivan, and T. Makimura, Opt. Express 21, 31837 (2013). 